\title{
MALIGNANT TRANSFORMATION IN THE COURSE OF A DENTIGEROUS CYST: A PROBLEM FOR A CLINICIAN AND A PATHOlOgist. CONSIDERATIONS BASED ON A CASE REPORT
}

\author{
Agnieszka Zapaea-Pośpiechi1, Grażyna Wyszyńska-Pawelec ${ }^{2}$, Dariusz AdameK ${ }^{3}$, \\ Romana Tomaszewska ${ }^{3}$, Maegorzata Zaleska ${ }^{1}$, Jan Zapala ${ }^{2}$
}

\author{
${ }^{1}$ Department of Oral Surgery, Jagiellonian University Medical College, Krakow, Poland \\ 2Department of Cranio-Maxillofacial Surgery of the Jagiellonian University, Krakow, Poland \\ 3 Department of Pathology, Jagiellonian University Medical College, Krakow, Poland
}

\begin{abstract}
Primary intraosseous squamous cell carcinoma (PIOSCC) is a rarely reported neoplasm resulting from malignant transformation of a dentigerous cyst of the mandible or maxilla. Until 2010, only 116 cases had been described. The diagnosis of PIOSCC is difficult because of non-specific symptoms. A case of a 66-year-old patient with PIOSCC arising from a dentigerous cyst of the mandible is presented. Both pathologists and clinicians should be aware of the probability of malignant transformation of dentigerous cysts during the two-stage treatment. The patient should be subject to regular clinical and radiographic examination.
\end{abstract}

Key words: primary intraosseous carcinoma, squamous cell carcinoma, dentigerous cyst, immunochemistry, radiography.

\section{Introduction}

Primary intraosseous squamous cell carcinoma (PIOSCC) is a rarely reported neoplasm arising in the mandible or maxilla from residual odontogenic epithelium, initially without contact with the oral mucosa. Diagnosis of PIOSCC might be made only when primary squamous cell carcinoma (SCC) of the oral mucosa is excluded as well as SCC of the paranasal sinuses or skin and metastatic tumours from malignant neoplasms localized in distant sites. There are three subtypes of PIOSCC: 1 - solid SCC, 2 - SCC arising from the lining of a dentigerous cyst, and 3-SCC from a keratocystic odontogenic tumour (KCOT) or other benign epithelial odontogenic tumours. The second subtype is the most common (over 50\% of cases) [1-5]. Primary intraosseous squamous cell carcinoma is more common in male patients over 60 years old [6-8]. Clinical symptoms, such as pain, oedema, inferior alveolar nerve paraesthesia, mobility of teeth, trismus, and mobility of fragments in pathological fracture of the mandible, are not specific for PIOSCC and might also occur in suppurative cysts $[2,5,9]$. As jaw cysts are often qualified for two-stage treatment (decompression followed by extirpation), especially in cases where immediate extirpation might cause pathological fracture, injury of teeth or other anatomical structures, the possibility of chronic inflammation-induced carcinogenesis should be taken into consideration. Especially, damage of DNA, apoptosis of normal cells, insertion of oncogenes and immunosuppression are mentioned as the causes of malignant transformation [10-13]. We present a case of PIOSCC arising from a dentigerous cyst of the mandible with a review of the literature concerning this uncommon malignancy.

\section{Case report}

A 66-year-old male patient was referred to the Outpatient Department of Oral Surgery of the Jagiellonian University in Kraków, due to painful inflammatory infiltration of the left perimandibular region of 2 days' 
duration. Medical history of the patient revealed the presence of a mandibular cyst diagnosed in his youth after a tooth extraction. However, the patient had never undergone treatment of the cyst. Comorbidities included diabetes type II, hypertension, gastric ulcer and spondyloarthrosis. On admission, extraoral examination revealed left facial oedema and enlarged lymphatic nodes of the IB level of the same site. Intraorally, bony expansion of the left mandibular shaft and purulent fistula in the area of tooth 36 were observed, whereas on palpation, crepitation in this region was found. A panoramic radiograph showed a unilocular, regular, radiolucent lesion extending from the area of tooth 41 to the area of tooth 36. The left inferior alveolar canal was displaced downwards (Fig. 1A). In local anaesthesia, an intraosseous biopsy of the lesion was performed. After incision of the oral mucosa, the thinned bone covering a cystic tumour filled with pus was exposed
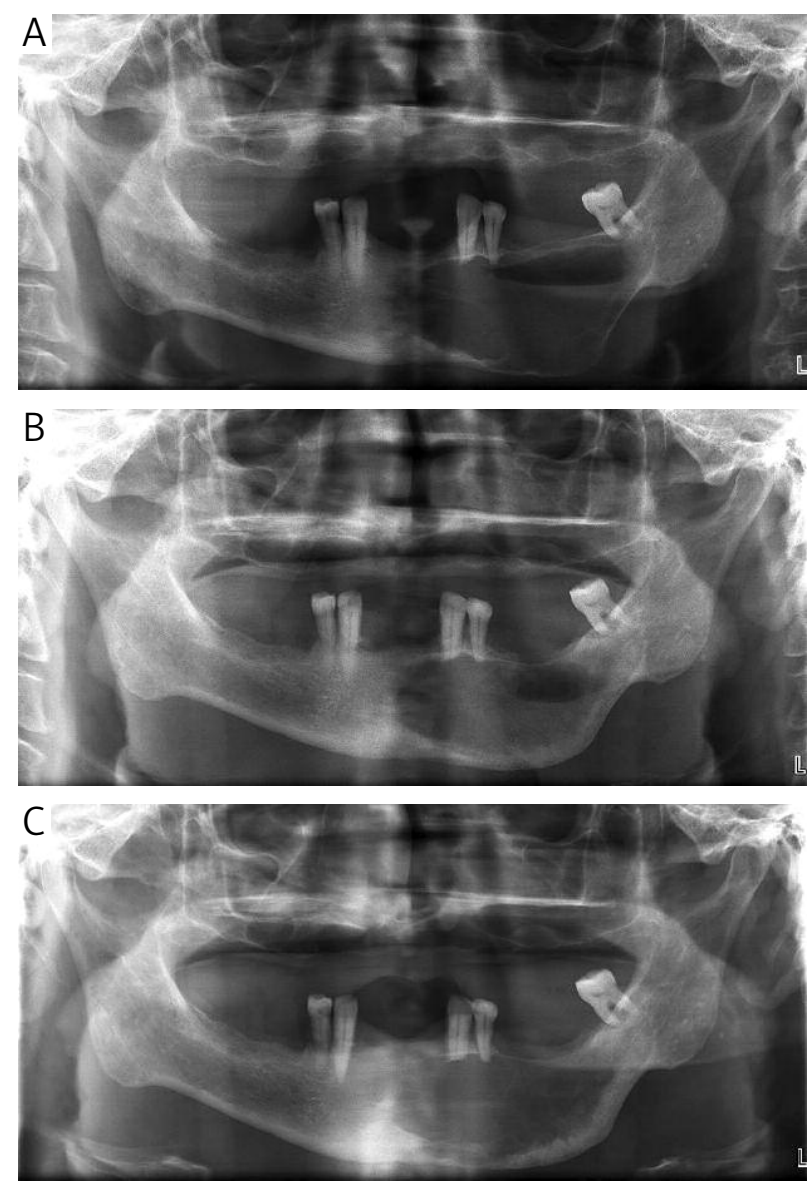

Fig. 1. A - Panoramic radiograph before treatment shows a unilocular, regular radiolucent lesion extending from the area of the mandibular right medial incisor (41) to the area of the mandibular left first molar (36). B - Control panoramic radiograph performed 3 months after surgery shows reduced cyst size and mild bone regeneration of the lower mandibular border, C - Panoramic radiograph performed 6 months after surgery shows irregular outline of the cystic lesion with bone infiltration especially in the lower border of the mandible, suggesting malignization
(Fig. 2). The harvested specimen consisted of a small bone fragment and part of the cyst wall. Histological examination of the specimen from the cyst wall revealed the presence of non-keratinized stratified squamous epithelium focally surrounded by an extensive outgrowth of unspecific granulation tissue with purulent and chronic inflammatory infiltrate. The material was fragmented - some fragments of the epithelium were detached from the rest of the tissue and some were intimately bound to the granulomatous outgrowth and even "embedded" within it (Fig. 3A). As a result, the whole histopathological picture was generally consistent with dentigerous cyst. Due to the possibility of pathological fracture of the mandible, two-stage treatment with cyst decompression and obturator as the first procedure was administered. The control panoramic radiograph performed 3 months following the surgery showed reduced cyst size and mild bone regeneration of the lower mandibular border (Fig. 1B). Although regular control examinations were administered, the patient appeared after 3 months presenting painful swelling of the left perimandibular area with enlargement of the regional lymph nodes. Intraorally, marked mobility of teeth 33 and 34 with purulent discharge from the gingival pockets was noted. A panoramic radiograph revealed irregular outline of the cystic lesion, especially in the lower border of the mandible where bone destruction was observed (Fig. 1C).

The second biopsy revealed the presence a tumoural mass (Fig. 4A). The histopathological examination showed unequivocally malignant, i.e. carcinomatous infiltration and, as a result, keratinizing SCC was diagnosed (Fig. 3B). The patient was immediately referred to the Department of Cranio-Maxillofacial Surgery of the Jagiellonian University in Kraków. Performed computed tomography (CT) of the head and neck revealed widening of the external outline of the anterior and lateral left part of the mandibular shaft with intraosseous osteolytic defects as well as infiltration of external muscles of the tongue and soft tissues in the mental region, along with

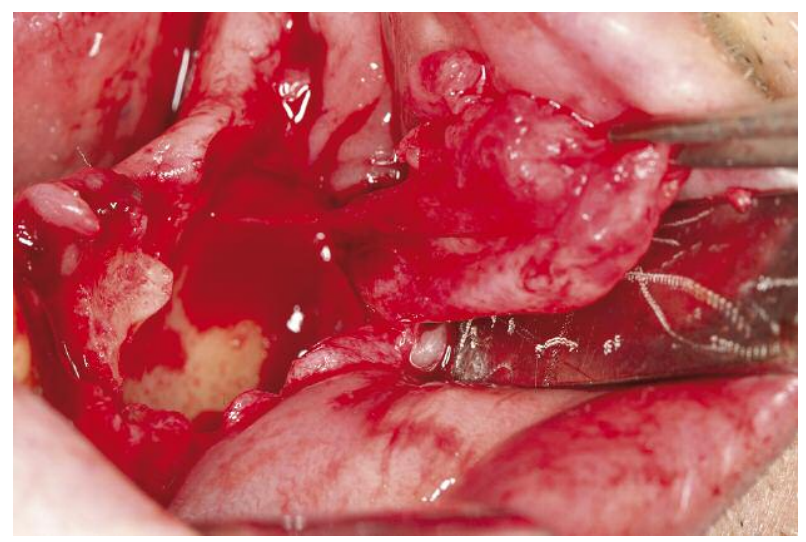

Fig. 2. Intraoperative view - decompression, extensive bony defect and destruction of the vestibular bone are visible 

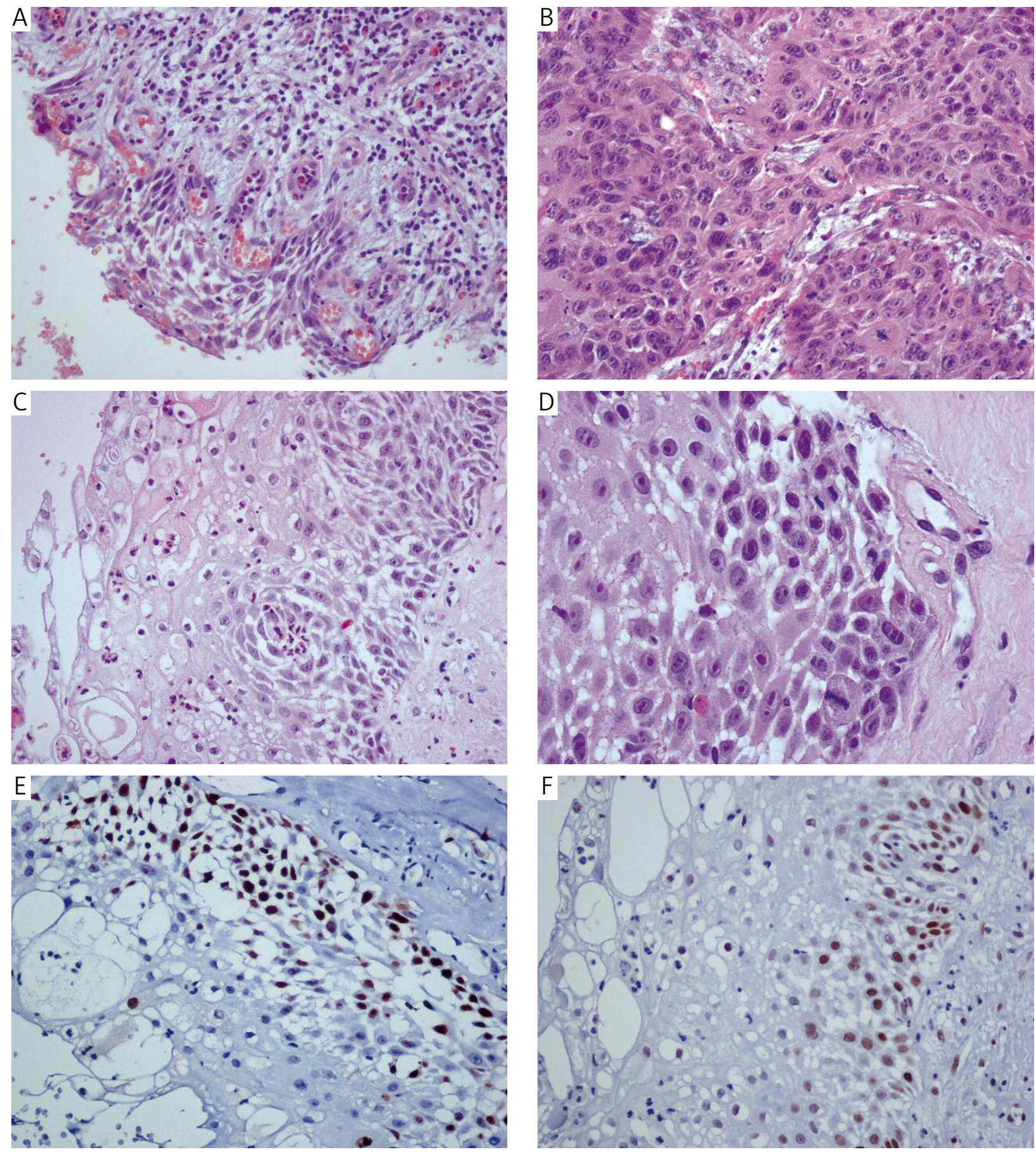

Fig. 3. A - Fragment of epithelial lining of dentigerous cyst. Noteworthy is intensive inflammatory response in the form of nonspecific granulation with mononuclear and suppurative infiltration intimately coupled with remnants of epithelium of which only more basic layers of cells remained. B - poorly differentiated squamous cell carcinoma arising in the dentigerous cyst. Numerous mitoses, including abnormal ones, are seen (material from second biopsy - all other microphotographs document findings from first biopsy) (HE, objective magnification $20 \times$ ). C - The epithelium of the dentigerous cyst shows marked degenerative changes with significant vacuolation of cells and the presence of numerous inflammatory cells permeating all layers of epithelium (HE, objective magnification $20 \times$ ). D - A close-up view of the pathologically changed epithelium of dentigerous cyst. A striking finding is the markedly enlarged nucleoli. Two mitoses are shown. However, the maturation of cells is preserved ( $\mathrm{HE}$, objective magnification $40 \times$ ). $\mathbf{E}-$ Epithelium of the dentigerous cyst - elevated proliferation activity emphasized by high number of cell nuclei immunopositive for Ki67 present not only in the basal region of epithelium but also in higher positioned cells. However, one has to have in mind that to some degree it might be a pseudomultiplication of $\mathrm{Ki} 67+$ nuclei due to tangential section of epithelium in this picture (immunostaining with antibody against Ki67, objective magnification $20 \times$ ). F - Marked immunopositivity for p53 protein - the general characteristic pattern of staining is very similar to that of Ki67 (immunostaining with antibody against p53, objective magnification $20 \times$ ) 

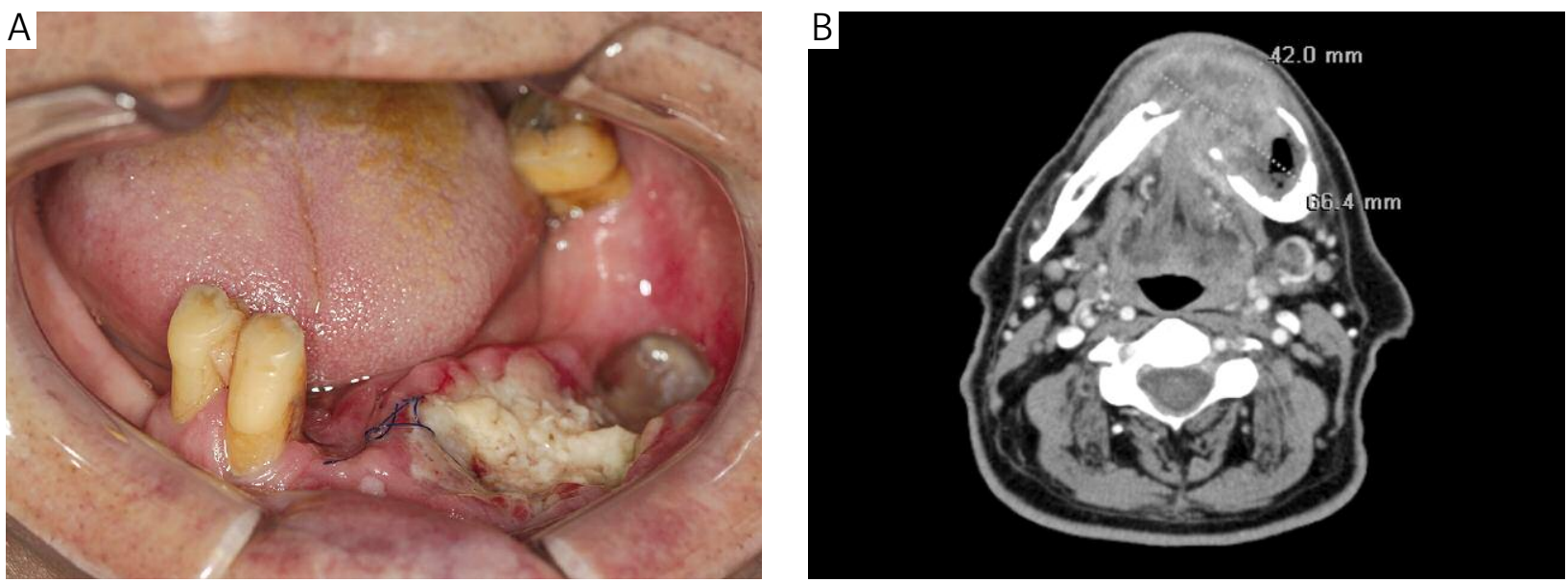

Fig. 4. A - Second biopsy - control examination 3 days after surgery; aggressive growth of the tumour can be seen.

B - CT scan showing osteolytic defects of the mandible along with infiltration of the external muscles of the tongue and soft tissues in the mental region

multiple pathological lymph nodes $(9-20 \mathrm{~mm}$ in diameter) at levels IA and IB bilaterally and level III on the right side (Fig. 4B). No other primary or metastatic tumours were diagnosed. Due to the necessity of segmental mandibulectomy of the mental part, tracheotomy was performed. Bilateral supraomohyoid neck dissection (levels III-I) by apron approach, followed by partial mandibulectomy in monobloc fashion, was carried out. Intraoperative histological examination of margins of the soft tissue resection was negative. The defect was reconstructed by a lingual flap. The wound healing was uneventful. Nasal tube feeding was carried out. The patient was discharged after decannulation. Histological examination of the entire specimen revealed moderately differentiated, partially keratinizing SCC and intraorally the resection was radical. However, metastatic tumours were present in 6 lymph nodes (at left IA, IB, right IB levels). Neck metastasis was an indication for radiation therapy, which is being carried out at present. Regarding the history of the disease, the histological material from the first biopsy was meticulously reexamined. Moreover, extra cut slides from the remaining material in the paraffin block were prepared and stained with hematoxylin-eosin and additionally immunohistochemically with antibodies against Ki67 and $\mathrm{p} 53$. Special attention was paid to any traces of changes which could indicate the existence of signs of malignant transformation in progress within the epithelium of the dentigerous cyst. In some of the fragments of the epithelial lining of the cyst, conspicuous changes such as focal epithelial irregularities of cell maturation, the presence of improperly larger nuclei, and markedly enlarged nucleoli were found (Fig. 3C,D). There were mitoses not only at the precise basal layer of epithelium but also at a somewhat separated position from the basal lamina. Immunostaining with antibodies against $\mathrm{Ki} 67$ and $\mathrm{p} 53$ also indicated elevated proliferation activity and immunopositivity in nuclei even in the upper half of the epithelium (Figs. 3E, F). In these regions of the above- mentioned changes, the epithelium also showed marked degenerative changes with significant vacuolation of cells, and the presence of numerous inflammatory cells permeating all layers of epithelium. Evidently, and what must be specially stressed, nowhere in the material from the first biopsy were there even any traces of a true carcinoma found, either in already examined slides or in slides additionally cut during re-examination of primary biopsy material.

\section{Discussion}

Primary intraosseous squamous cell carcinoma arising from a dentigerous cyst is a very rare malignant tumour. Until 2010 only 116 cases had been reported [5]. Primary intraosseous squamous cell carcinoma constitutes $1-2.5 \%$ of all odontogenic tumours $[14\}$ and, according to van der Waal [15], 1-2\% of all intraoral cancers. The tumour is localized almost exclusively in the jaws and arises from the remnants of odontogenic epithelium [4]. Chronic inflammation might be the main factor leading to malignant transformation of the dentigerous cyst's epithelium, as was probably the case in our patient $[10,12]$. The presented case meets the diagnostic criteria of PIOSCC $[4,16]$ : a) initially the tumour had no connection with the oral mucosa, b) histological examination confirmed diagnosis of SCC, c) diagnostic examinations ruled out the possibility of metastasis from a distant primary tumour in a 6-month period. Gardner's histological criteria [17] required for diagnosis of the second subtype of PIOSCC also include confirmation of a transitional area between typical epithelium of the cyst and infiltration of SCC, absence of carcinomatous infiltration in the overlying epithelium and absence of another focus of SCC in the surrounding tissues. Although the presented case fulfils the second and third criterion, histological examination did not reveal the presence of a transitional area (in samples from the 2 nd biopsy there was only carcinomatous infiltra- 
tion). On the other hand, the re-examination of the first biopsy material showed signs of dysplasia within epithelium of the dentigerous cyst. Moreover, the prolonged course of the disease, its clinical features and characteristic radiographic symptoms, changing during treatment (intraosseous cyst-like lesion, appearance of irregular margins followed by bone destruction) [18], may suggest malignant transformation of the dentigerous cyst. From the diagnostic histopathological point of view the presented case emphasizes the need to pay special attention to the status of epithelium lining dentigerous cysts. In the first biopsy obviously there was no evidence and no reasons to recognize carcinoma; however, as described above, there were some evident signs of dysplasia of the cyst epithelium. The epithelial changes within a dentigerous cyst with a strong inflammatory process have a reactive character. Nevertheless, this case shows that dysplastic changes may sometimes occur and then they necessitate undertaking appropriate modification of the approach to treatment. In advanced cases of PIOSCC, misleading clinical symptoms might suggest a diagnosis of cancer of the oral mucosa infiltrating the mandible. Primary intraosseous squamous cell carcinoma is usually a well- or moderately differentiated cancer (about $85 \%$ of cases) [19], as it is in our case. Regional metastases in the second subtype of PIOSCC are uncommon and in Bodner's meta-analysis [5] only 6 cases are mentioned. However, in our patient, multiple bilateral cervical metastases were found. Surgical treatment is the method of choice in patients with PIOSCC $[5,6,19]$ but might also be combined with radiation therapy, as in the current case. Prognosis of patients with PIOSCC is poor; the overall survival rate at 5 years ranges from $30 \%$ to $40 \%$ [20].

\section{Conclusions}

As the treatment of extensive dentigerous cysts usually comprises a two-stage procedure, during the entire, prolonged time of therapy, the probability of malignant transformation of the cyst's epithelial lining should be taken into consideration. In consequence, the patients require regular clinical and radiographic control as well as immediate histological verification in unclear cases. Pathologists should be especially cautious and critical when recognizing and interpreting the changes within the epithelia of a dentigerous cyst, not only with regard to the differentiation from KCOT and the unicystic ameloblastoma, but also in connection with possible cancerization of the dentigerous cyst.

\section{The authors declare no conflict of interest.}

\section{References}

1. Suei Y, Taguchi A, Tanimoto K. Recommendation of modified classification for odontogenic carcinomas. Med Hypotheses 2004; 62: 382-386
2. Waldron CA, Mustoe TA. Primary intraosseous carcinoma of mandible with probable origin in an odontogenic cyst. Oral Surg Oral Med Oral Pathol 1989; 67: 716-724.

3. Hwang EH, Choi YS, Lee SR. Primary intraosseous carcinoma of the mandible. Korean J Oral Maxillofac Radiol 2005; 35: 235-239.

4. Sengupta S, Vij H, Vij R. Primary intraosseous carcinoma of the mandible: A report of two cases. J Oral Maxillofac Pathol 2010; 14: 69-72.

5. Bodner L, Manor E, Shear M, et al. Primary intraosseous squamous cell carcinoma arising in an odontogenic cyst- a clinicopathologic analysis of 116 reported cases. J Oral Pathol Med 2011; 40: 733-738.

6. Thomas G, Pandey M, Mathew EK, et al. Primary intraosseous carcinoma of the jaw: pooled analysis of world literature and report of two new cases. Int J Oral Maxillofac Surg 2001; 30: 349-355.

7. Charles M, Barr T, Leong I, et al. Primary intraosseous malignancy originating in an odontogenic cyst in a young child. J Oral Maxillofac Surg 2008; 66: 813-819.

8. Huang JW, Luo HY, Li Q, et al. Primary intraosseous carcinoma of the jaws. Clinicopathologic presentation and prognostic factors. Arch Pathol Lab Med 2009; 133: 1834-1840.

9. Shambhulingappa P, Sheikh S, Puri N, et al. Primary intraosseous carcinoma of mandible: An update on review of literature with a case report. J Clin Exp Dent 2010; 2: 91-95.

10. Coussens LM, Werb Z. Inflammation and cancer. Nature 2002; 420: 860-867.

11. Choi S, Myers JN. Molecular pathogenesis of oral squamous cell carcinoma: implication for therapy. J Dent Res 2008; 87: 14-32.

12. Karin M, Greten FR. NF-kappa B: linking inflammation and immunity to cancer development and progression. Nat Rev Immunol 2005; 5: 749-759.

13. Hold GL, El-Omar ME. Genetic aspects of inflammation and cancer. Biochem J 2008; 410: 225-235.

14. Matsuzaki H, Karase N, Matsumura T, et al. Solid-type intraosseous squamous cell carcinoma of the mandible: a case report with histopathological and imaging features. Oral Maxillofac Radiol 2012; 114: 71-77.

15. Van der Waal I, Rauhamaa R, van der Kwast WA, et al. Squamous cell carcinoma arising in the lining of odontogenic cysts. Report of 5 cases. Int Oral Surg 1985; 14: 146-152.

16. Murillo-Cortes J, Etayo-Perez A, Sebastian-Lopez C, et al. Primary intraosseous carcinoma arising in a mandibular cyst. Medicina Oral 2002; 7: 370-374.

17. Gardner AF. A survey of odontogenic custs and their relationship to squamous cell carcinoma. J Can Dent Assoc 1975; 41: 161-167.

18. Cavalcanti MG, Veltrini VC, Ruprecht A, et al. Squamous-cell carcinoma arising from an odontogenic cyst-The importance of computed tomography in the diagnosis of malignancy. Oral Med Oral Pathol Oral Radiol Endod 2005; 100: 365-368.

19. Chaisuparat R, Coletti D, Kolokythas A, et al. Primary intraosseous odontogenic carcinoma arising in an odontogenic cyst or de novo: A clinicopathologic study of six new cases. Oral Med Oral Pathol Oral Radiol Endod 2006; 101: 194-200.

20. Lugakingira M, Pytynia K, Kolokythas A, Miloro M. Primary intraosseous carcinoma of the mandible: case report and review of the literature. J Oral Maxillofac Surg 2010; 68: 2623-2629.

\section{Address for correspondence}

Agnieszka Zapała-Pośpiech

Department of Oral Surgery

Jagiellonian University Medical College

Montelupich 4

31-155 Kraków, Poland

tel. +48124245468

fax +48124245499

e-mail: agnieszka.zapala-pospiech@uj.edu.pl 